IN PRACTICE

\title{
The response to sertraline in men with chronic pelvic pain syndrome
}

\author{
R A Lee, R M West, J D Wilson
}

Sex Transm Infect 2005;81:147-149. doi: 10.1136/sti.2004.010868

\begin{abstract}
See end of article for authors' affiliations

....................

Correspondence to: R A Lee, Department of Genitourinary Medicine, The General Infirmary at Leeds, Great George Street, Leeds LSI 3EX, UK; rachel.lee@leedsth.nhs.uk

Accepted for publication 23 August 2004
\end{abstract}

n 1995 the National Institutes of Health reclassified men with prostatitis-type symptoms into four main categoriesacute bacterial prostatitis, chronic bacterial prostatitis, chronic pelvic pain syndrome inflammatory (inflammatory cells but no bacteria found in prostatic fluid), and chronic pelvic pain syndrome non-inflammatory (neither inflammatory cells nor bacteria identified in prostatic fluid). ${ }^{1}$ The four glass test described by Meares and Stamey in 1968 is used to distinguish between chronic bacterial prostatitis and the chronic pelvic pain syndromes. ${ }^{2}$ It can also distinguish between inflammatory and non-inflammatory chronic pelvic pain syndrome (CPPS) although this distinction is not particularly useful as they are almost identical both in clinical presentation and response to treatment. Therefore, in our study both subdivisions of CPPS were included.

The aetiology of CPPS remains unclear with various theories suggested including the urodynamic theory in which it is thought that a high resting tone of the external urethral sphincter leads to the reflux of urine into the prostate gland during micturition resulting in a chemical prostatitis. ${ }^{3}$ Other theories include pelvic floor tension myalgia, ${ }^{4}$ pelvic sympathetic nervous system abnormalities, ${ }^{5}$ pelvic venous congestion, ${ }^{6}$ and interstitial cystitis. ${ }^{7}$ It has also been suggested that there is a significant psychological component to this condition with one study demonstrating that $62 \%$ with CPPS had elevated scores for depression compared with $16 \%$ of controls. $^{8-11}$

Patients present with chronic genito-pelvic pain, which is often accompanied by urinary dysfunction, ejaculatory disturbance, or psychosexual problems. ${ }^{12}$ These symptoms are distressing and often highly debilitating for patients. Currently there are no universally effective treatments available making it an extremely frustrating condition to manage for both physician and patient alike.
Antidepressants have been effectively used for the treatment of other chronic pain syndromes and tricyclic antidepressants are commonly used for chronic genital pain in women. ${ }^{13}{ }^{14}$ To date there have been no studies performed using antidepressants in men with CPPS. We chose to perform such a study using sertraline (a serotonin specific reuptake inhibitor, SSRI) as we thought that the anticholinergic side effects of the tricyclic antidepressants might make them a less favourable choice for a placebo controlled trial.

\section{METHODS}

Men between the ages of 18-65 were evaluated clinically and by the use of a validated prostatitis symptom questionnaire and the American Urologists Association (AUA) benign prostatic hyperplasia questionnaire. ${ }^{15}$ Those with symptoms compatible with CPPS underwent the four glass test to exclude bacterial prostatitis. Urethral swabs were taken for microscopy and culture for Neisseria gonorrhoeae. A first void urine sample was sent for polymerase chain reaction (PCR) for Chlamydia trachomatis. Serum samples were taken for prostate specific antigen, urea, and electrolytes.

Patients with urethritis, symptoms suggestive of benign prostatic hyperplasia or significant abnormalities on baseline bloods were excluded. Other exclusion criteria were current treatment with an antidepressant or anxiolytic drug, history of seizures, or any history of hypersensitivity or intolerance to an SSRI.

Abbreviations: CPPS, chronic pelvic pain syndrome; HAD scale, Hospital Anxiety and Depression scale; PCR, polymerase chain reaction; PSex questionnaire, psychosexual questionnaire; PSF, prostatic symptom frequency; PSS, prostatic symptom severity; SSRI, serotonin specific reuptake inhibitor 
Table 1 Summary of results at baseline and at 13 weeks

\begin{tabular}{|c|c|c|c|c|c|c|}
\hline \multirow[b]{2}{*}{ Mean score } & \multicolumn{2}{|l|}{ Baseline } & \multirow{2}{*}{$\begin{array}{l}\text { Mann-Whitney } U \text { test } \\
\text { p value }\end{array}$} & \multicolumn{2}{|l|}{13 weeks } & \multirow{2}{*}{$\begin{array}{l}\text { Mann-Whitney } U \text { test } \\
\text { p value }\end{array}$} \\
\hline & Active drug & Placebo & & Active drug & Placebo & \\
\hline PSS & 23.4 & 28.0 & 0.94 & 17.3 & 26.0 & 0.34 \\
\hline PSF & 15.9 & 18.7 & 0.89 & 12.3 & 17.7 & 0.09 \\
\hline HADa & 7.6 & 8.2 & 0.52 & 6.7 & 5.6 & 0.40 \\
\hline HADd & 4.7 & 4.5 & 0.52 & 3.1 & 3.8 & 0.30 \\
\hline PSex & 22.6 & 20.9 & 0.63 & 22.1 & 23.1 & 0.44 \\
\hline
\end{tabular}

PSS, prostatic symptom severity; PSF, prostatic symptom frequency; HAD, Hospital Anxiety and Depression scale; HADd, HAD depression; PSex, psychosexual questionnaire.

All participants gave written consent and ethical approval had been obtained. Four baseline questionnaires were completed including a validated prostatic symptom frequency (PSF) and prostatic symptom severity (PSS) score, ${ }^{16}$ the Hospital Anxiety and Depression (HAD) scale, ${ }^{17}$ and a psychosexual (Psex) questionnaire. They were then randomised in a double blind fashion to receive either sertraline $50 \mathrm{mg}$ daily or matched placebo for 13 weeks. At 13 weeks both the subjects and investigator were unblinded and those receiving placebo were given the opportunity to cross over into the treatment arm for a further 13 weeks until the study end at 26 weeks. Subjects initially randomised to receive sertraline were given the option to continue this for the full 26 weeks. All subjects were assessed at 2 weeks for any adverse reaction to the treatment and again at 6, 13, and 26 weeks at which visits the four baseline questionnaires were again completed.

A Mann-Whitney U test was performed to compare scores in the sertraline versus placebo arms at baseline and at 13 weeks. The Wilcoxon signed rank test was used to compare change in scores of the two arms between baseline and 13 weeks. From 13 weeks all of the subjects received active treatment so the mean scores of all subjects at baseline were compared with the scores following 13 weeks of sertraline using the Wilcoxon signed rank test.

\section{RESULTS}

Fourteen subjects entered the trial and were randomised equally between the active and placebo arms. Results of the Mann-Whitney U test comparing baseline scores between the two study arms demonstrated no difference between the groups at baseline (table 1).

One subject withdrew from the study after 4 weeks because of adverse effects. He described feeling "spaced out" and was receiving placebo. Overall, there was no difference in reporting of adverse events between the placebo and sertraline arms.

To evaluate the effectiveness of sertraline compared to placebo we compared the scores between the two study arms at week 13 by the Mann-Whitney U test. There was no significant difference because of the small subject numbers. We also used the Wilcoxon signed rank test to compare the changes in mean scores from baseline to 13 weeks in the active and placebo groups. An improvement is denoted by a decrease in PSF, PSS, and HAD scores and an increase in the PSex score. At week 13 there was a mean reduction in PSS scores of 6.1 in the active and 2.0 in placebo group, and in PSF scores of 3.6 and 1.0, respectively. There was no statistically significant difference between the two groups but there was a trend towards sertraline being associated with an improvement in PSF, PSS, and HAD depression (HADd) scores (table 2).

After the unblinding at 13 weeks all six subjects receiving placebo chose to cross over to the sertraline arm so we have results from all 13 men following 13 weeks of treatment with sertraline. Analysing the results as a case series the scores at baseline were compared with those following 13 weeks of sertraline using the Wilcoxon signed rank test (table 3). There was a significant improvement in PSF and PSS over this 13 week period. There was also a non-significant improvement in HADd score.

\section{DISCUSSION}

Male chronic pelvic pain syndrome (CPPS) is a condition with significant morbidity that not infrequently presents to genitourinary medicine services. ${ }^{18}$ Its aetiology remains unclear with no universally effective treatments available, making it a difficult challenge for any clinician. Evidence for the effectiveness of the available treatments is lacking or based on small uncontrolled trials. Although male CPPS is not uncommon recruitment into trials is often poor in this group making it a difficult condition to study. Our trial was no exception as we have full data on only 13 men. A power calculation performed at the study planning stage suggested that if the difference of PSS plus PSF scores was 10 between the active and placebo groups at week 13, we would need to recruit 60 patients, 30 in each arm, to have $80 \%$ power to be able to show a significant difference. This means the randomised control trial was well underpowered. The main reasons given for declining entry into the trial were dissatisfaction with the possibility of randomisation to the placebo arm and reluctance to take an antidepressant drug. Several patients were excluded because of current antidepressant use.

Table 2 Mean change in scores between baseline and 13 weeks (sertraline versus placebo)

\begin{tabular}{lllll}
\hline \multicolumn{5}{l}{ Mean change in score from $\mathbf{0}$ to $\mathbf{1 3}$ weeks } \\
\cline { 2 - 5 } & Sertraline & p Value & Placebo & p Value \\
\hline PSF & -3.6 & 0.16 & -1.0 & 0.41 \\
PSS & -6.1 & 0.12 & -2.0 & 0.44 \\
HADa & -0.9 & 0.39 & -2.5 & 0.18 \\
HADd & -1.6 & 0.19 & -0.7 & 0.41 \\
Psex & -0.5 & 0.43 & +2.2 & 0.27 \\
\hline
\end{tabular}




\begin{tabular}{lcccc|}
\hline Table 3 & Mean change in score from baseline to 13 weeks of taking sertraline \\
\hline & $\begin{array}{l}\text { Baseline } \\
\text { (mean) }\end{array}$ & $\begin{array}{l}13 \text { weeks } \\
\text { (mean) }\end{array}$ & Mean change in score & p Value \\
\hline PSF & 17.2 & 11.3 & -5.9 & 0.03 \\
PSS & 25.5 & 13.8 & -11.7 & 0.01 \\
HADa & 7.8 & 5.7 & -2.1 & 0.14 \\
HADd & 4.6 & 2.4 & -2.2 & 0.07 \\
Psex & 21.8 & 24.1 & +2.3 & 0.17 \\
\hline
\end{tabular}

In 1999 the National Institutes of Health $(\mathrm{NIH})$ published their validated Chronic Prostatitis Symptom Index incorporating both a measure of symptoms and impact on quality of life. ${ }^{19}$ This is a superior tool for evaluating men with CPPS but as recruitment was already under way before its introduction it was not used in our study. The questionnaires used in our study had, however, been validated in previous prostatitis studies. ${ }^{16}$

Such a small study group makes interpretation of the results somewhat difficult. Comparing the change in mean scores from the prostatic symptom frequency (PSF), prostatic symptom severity (PSS), Hospital Anxiety and Depression (HAD), and psychosexual (Psex) questionnaires from baseline to 13 weeks there was a trend towards a greater improvement in PSF, PSS, and HAD depression (HADd) scores in the sertraline group when compared with placebo. However, these values did not reach statistical significance, which is not surprising given the small numbers involved. There was a non-statistically significant trend towards sertraline being associated with a deterioration in the psychosexual score, which was predictable, as loss of libido, erectile dysfunction, and delayed ejaculation are all recognised side effects of the SSRI antidepressant group.

Following unblinding we have scores on all 13 subjects after taking sertraline for 13 weeks. Comparing the scores at baseline, results after 13 weeks treatment showed a statistically significant improvement in PSF and PSS, and a nonsignificant improvement in the HAD depression scores. This suggests that sertraline is effective in reducing the frequency and severity of symptoms in men with CPPS. However, these results include men known to be on active therapy so a placebo effect cannot be excluded.

In our study sertraline was well tolerated. The SSRI antidepressants have minimal interactions reported with other drugs and if truly effective would be a welcome addition to the somewhat poor repertoire of treatments available to men with CPPS. The results from this study are encouraging but we cannot exclude a placebo effect. It is clear that similar larger studies using the NIH Chronic Prostatitis Symptom Index as an outcome measure are required in order to further evaluate the use of antidepressants in men with CPPS.

\section{ACKNOWLEDGEMENTS}

We thank the MSSVD for funding the manufacture of the matched placebo used in this study.

\section{CONTRIBUTORS}

RL coordinated the study, collected the data, and was responsible for the first draft of the manuscript; RW and JW performed the data analysis; the study was designed by JW; all authors read and contributed to the production of the paper.

\section{Authors' affiliations}

R A Lee, J D Wilson, Department of Genitourinary Medicine, The General Infirmary at Leeds, UK

R M West, Nuffield Institute for Health, University of Leeds, UK

Conflict of interest: None.

\section{REFERENCES}

1 NIH Workshop on Chronic Prostatitis. Executive summary. Bethseda, MD, 1995 (www.niddk.nih.gov./health/urolog/pubs/cpwork/cpwork.htm).

2 Meares EM, Stamey TA. Bacterial localisation patterns in bacterial prostatitis and urethritis. Invest Urol 1968;5:492-518.

3 Barbalias GA. Prostatodynia or painful male urethral syndrome? Urology 1990;36:146-53.

4 Segura JW, Opitz JL, Greene LF. Prostatosis, prostatitis or pelvic floor tension myalgia. J Urol 1979;122:168-9.

5 Barbalias GA, Meares EM, Sant GR. Prostatodynia: clinical and urodynamic characteristics. J Urol 1983;130:514-17.

6 Terasaki T, Watanabe $H$, Saitoh $M$, et al. Magnetic resonance angiography in prostatodynia. Eur Urol 1995;27:280-285.

7 Miller JL, Rothman I, Bavendan TG, et al. Prostatodynia and interstitial cystitis: one and the same? Urology 1995:45:587-90.

8 De la Rosette JJMCh, Ruijgrok MCM, Jeuken JMG, et al. Personality variables involved in chronic prostatitis. Urology 1993;42:654-62.

9 Wenninger K, Heiman JR, Rothman I, et al. Sickness impact of chronic nonbacterial prostatitis and its correlates. J Urol 1996;155:965-8.

10 Egan KJ, Krieger JN. Psychological problems in chronic prostatitis patients with pain. Clin J Pain 1994;10:218-26.

11 Berghuis JP, Heiman JR, Rothman I, et al. Psychological and physical factors involved in chronic idiopathic prostatitis. J Psychosom Res 1996;41:313-25.

12 Krieger JN, Egan KJ, Ross SO, et al. Chronic pelvic pains represent the most prominent urogenital symptoms of "chronic prostatitis". Urology 1996;48:715-22

13 Jung AC, Staiger T, Sullivan M. The efficacy of selective serotonin reuptake inhibitors for the management of chronic pain. J Gen Intern Med 1997; 12:384-9.

14 McKay M. Dysesthetic ('essential') vulvodynia. Treatment with amitriptyline. $J$ Reprod Med 1993;38:9-13.

15 Barry MJ, Fowler FJ, O'Leary MP, et al. The American Urological Association symptom index for benign prostatic hyperplasia. J Urol 1992; 148:1549-57.

16 Nickel JC, Sorenson R. Transurethral microwave thermotherapy for nonbacterial prostatitis: a randomised double-blind sham controlled study using new prostatitis specific assessment questionnaires. J Urol 1996;155:1950-5.

17 Zigmund AS, Snaith RP. The Hospital Anxiety and Depression Scale. Acta Psychiatr Scand 1983;67:361-70.

18 Luzzi GA, Bignell C, Mandal D, et al. Chronic prostatitis/chronic pelvic pain syndrome: national survey of genitourinary medicine clinics. Int J STD AIDS 2002;13:416-19.

19 Litwin MS, McNaughton-Collins M, Fowler FJ, et al. The National Institutes of Health Chronic Prostatitis Symptom Index: development and validation of a new outcome measure. J Urol 1999;162:369-75. 